\title{
Label-free bioanalysis of Leishmania infantum using refractive index tomography with partially coherent illumination
}

\author{
Juan M.Soto*1 | José A. Rodrigo ${ }^{1}$ | Alicia Mas² | Gustavo Domínguez-Bernal ${ }^{2}$ | Tatiana Alieva ${ }^{1}$
}

${ }^{1}$ Department of Optics, Faculty of Physical Sciences, Complutense University of Madrid, Madrid, Spain

${ }^{2}$ Department of Animal Health, Faculty of Veterinary Science, Complutense

University of Madrid, Madrid, Spain

\section{Correspondence}

*Juan M.Soto, Complutense University of Madrid, 28040 Madrid, Spain. Email:

juansoto@ucm.es

\begin{abstract}
In this work we report the use of refractive index (RI) tomography for quantitative analysis of unstained DH82 cell line infected with Leishmania infantum. The cell RI is reconstructed by using a modality of optical diffraction tomography technique that employs partially coherent illumination, thus enabling inherent compatibility with conventional wide-field microscopes. The experimental results demonstrate that the cell dry mass concentration (DMC) obtained from the RI allows for reliable detection and quantitative characterization of the infection and its temporal evolution. The RI provides important insight for studying morphological changes, particularly membrane blebbing linked to an apoptosis (cell death) process induced by the disease. Moreover, the results evidence that infected DH82 cells exhibit a higher DMC than healthy samples. These findings open up promising perspectives for clinical diagnosis of Leishmania.
\end{abstract}

\section{KEYWORDS:}

optical microscopy, optical properties, refractive index, optical tomography, partially coherent illumination, scattering measurement

\section{1 | INTRODUCTION}

Optical diffraction tomography (ODT) is a computational imaging technique that allows reconstructing the specimen 3D refractive index (RI) distribution. The RI has been widely adopted for obtaining quantitative biological information such as the shape, density, volume, dry and wet mass and chemical composition of cells [1-5]. ODT provides the label-free study of the sample without using any chemical marker or fluorescent tagging, thus avoiding photobleaching or modifying its behaviour. Thanks to its noninvasive nature, the 3D RI analysis has been recently exploited in several biomedicine applications. For instance, in Ref. 6 RI tomograms have been applied to find morphological and behavioral differences between diabetic and non-diabetic red blood cells. It has also led to promising results in cancerous epithelial cells bioanalysis [7] and influenza virus presence in human alveolar epithelial cells ${ }^{[8}$. In this way, RI changes have even been considered a biomarker for the monitorization and diagnosis of certain diseases, such as malaria infection induced by the Plasmodium falciparum parasite and detected by blood screening [910].

Most implementations of ODT available in the literature apply a coherent laser source for illuminating the sample, see for example [11][13]. Nevertheless, it has been recently demonstrated the benefits of using partially coherent and quasi-monochromatic illumination (e.g., halogen or LED lamp) for ODT (referred to as PC-ODT) providing speckle-free imaging [14-16]. Here we consider the PC-ODT technique reported in [15]16 that allows for straightforward 3D label-free imaging at video-rate $(10 \mathrm{fps}$, a rate about $5 \times$ faster than in C-ODT with similar spatial resolution). The experimental setup comprises an affordable high-speed 
optical scanning system enabling compatibility with commercial wide-field microscopes. These advantages of PC-ODT encourage its application in 3D analysis of living cells, in particular for the monitorization and diagnosis of certain diseases.

In this work, for the first time, we apply this PC-ODT technique for 3D RI imaging and detection of Leishmania parasites in DH82 cell line (blood macrophages) for a better understanding of the disease spreading in host cells. Leishmaniosis is a vector-borne zoonosis widely distributed throughout the world whose responsible etiological agent is a protozoan belonging to the genus Leishmania with several species according to their geographical distribution [17]. Leishmania is a dimorphic parasite with an intracellular phase called amastigote, and an extracellular flagellated phase known as promastigote. The promastigotes need over a period of 4 to $24 \mathrm{~h}$ in order to transform into amastigotes. Thereafter, the infection is sustained by amastigote forms in the infected hosts. The sizes of these intracellular amastigotes typically measures $1-5 \mu \mathrm{m}$ in length by $1-2 \mu \mathrm{m}$ in width. Vector transmission is carried out from a diptera known as Sandfly and the main species affected are human and canines, whereas more than 50 species have been described worldwide as reservoirs [18].

The study of Leishmania infection has attracted great attention from the scientific community because, according to the World Health Organization, 20000 people die annually from this disease [19]. To date, there is still no vaccine on the market capable of preventing human leishmaniasis, and although there are vaccines for veterinary use, their effectiveness is quite limited [20]. The antileishmanial treatments present important disadvantages such as the appearance of resistances and their high toxicity making necessary the development of new drugs [21]. Consequently, a tool that provides an accurate intracellular screening of the sample is needed and, in this regard, bioanalysis based on RI emerges as an attractive alternative to traditional staining techniques.

\section{2 | MATERIALS AND METHODS}

\section{1 | Sample description}

\subsection{1 | Cell culture preparation}

DH82 (ATCC R CRL-10389TM) is a macrophage cell line obtained from a dog with a neoplasm of histiocytic origin ${ }^{222}$. It was maintained in DMEM medium supplemented with $10 \%$ Foetal Bovine Serum (FBS), $100 \mathrm{U} / \mathrm{ml}$ penicillin and $100 \mu \mathrm{g} / \mathrm{ml}$ streptomycin. Then, it was incubated at $37^{\circ} \mathrm{C} 5 \% \mathrm{CO} 2$ in six-well plates.

Leishmania infantum BCN150 strain cells (M/CAN/ES/96/BCN150 zymodeme MON-1), previously reported in [23], were cultivated in Schneider's Insect Medium (Biowest, France) supplemented with $20 \%$ FBS, $100 \mathrm{U} / \mathrm{ml}$ penicillin and $100 \mathrm{mg} / \mathrm{ml}$ streptomycin at $26^{\circ} \mathrm{C}$ until the stationary phase and they were used to infect DH82 cells.

\subsection{2 | Infection protocol}

DH82 macrophages were cultured in 24-well plates overnight at a concentration of $1 \times 10^{6}$ cells per well. On the following day, promastigotes were added to the cells at a ratio 10:1 (parasites: cells) according to the protocol previously described in ${ }^{[24]}$. Cells were incubated at $37^{\circ} \mathrm{C} 5 \% \mathrm{CO}_{2}$ for 4 hours and, subsequently, extracellular parasites were eliminated by washing twice with Dulbecco' $s$ Phosphate Buffered Saline (DPBS) establishing the initial infection time point. Then, fresh medium was added, and cells were cultivated for $24 \mathrm{~h}$ and $72 \mathrm{~h}$. Finally, the cells were fixed using methanol $70 \%$ during 5 min at room temperature.

\subsection{3 | Staining and fixation of the samples}

The samples were divided into two groups according to whether they undergo staining or not. For those ones that were stained, each slide is immersed in $250 \mu \mathrm{l} 5 \%$ Giemsa's stain improved R66 solution Gurr and stored for 15 minutes at ambient temperature. After that, we washed five times each sample with distilled water and let it dry out. After the procedure of staining, the sample is fixed with methanol and all the cytoplasm water content has been replaced by the fixation medium. 


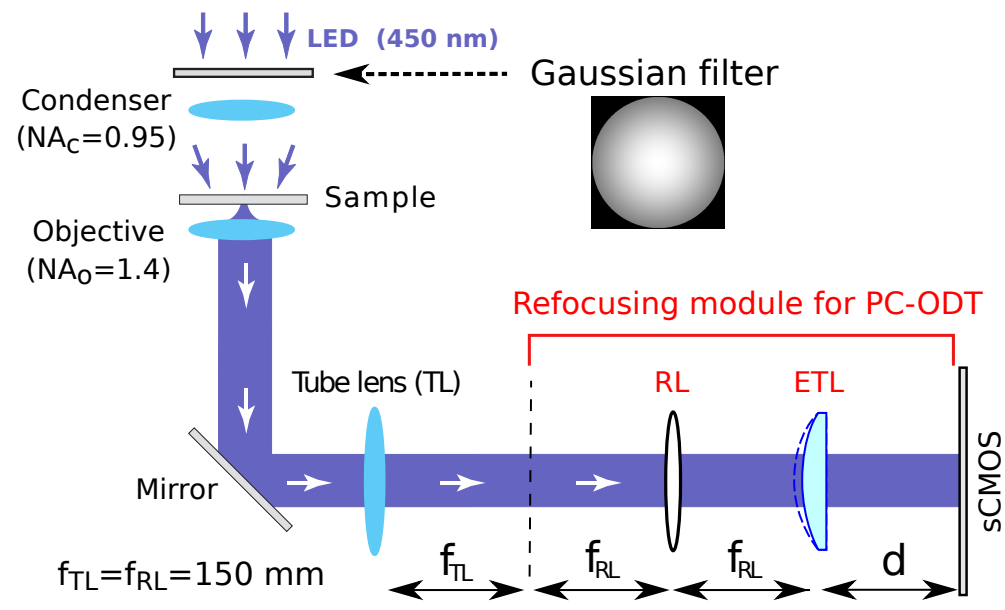

FIGURE 1 Sketch of the wide-field microscope including two high-NA lenses (condenser and objective) and a refocusing module comprising the lenses RL and ETL required for axial scanning in PC-ODT.

\section{2 | Tomographic reconstruction of the sample}

\subsection{1 | Experimental setup}

The 3D RI distribution $(n(\mathbf{r})$, where $\mathbf{r}=(x, y, z)$ denotes the spatial coordinates vector) of a transparent (weakly absorbing) sample was reconstucted by using the PC-ODT technique. Particularly, PC-ODT allows the RI reconstruction from the measurement of a through focus-series intensity images provided by a widefield microscope under partially coherent illumination. Here we applied the experimental setup reported in [15]16] consisting of a microscope equipped with a fast optical refocusing module as sketched in Fig. 1 in which the illumination is provided by a quasi-monochromatic LED source $\left(\lambda_{0}=450 \mathrm{~nm}\right)$. The microscope includes two high numerical aperture (NA) lenses, a condenser of $\mathrm{NA}_{\mathrm{c}}=0.95$ and an objective of $\mathrm{NA}_{\mathrm{o}}=$ 1.4 (100x magnification). The optical refocusing module comprises a relay lens (RL) and an electrically tunable lens (ETL) used for optical axial scanning of the sample, by modifying the focal length of the ETL. It is worth underlining that this experimental module can be easily included in a common wide-field microscope. We have also included a gaussian illumination (GI) instead of the conventional bright-field illumination (BFI). As demonstrated in [25], such a GI allows for proper collection of relevant object spatial frecuency content otherwise attenuated by the optical transfer function (OTF) of the microscope when BFI is applied. Here, as in Ref. [16], each measurement of the intensity stack of axially scanned images has a size of $400 \times 400 \times 50$ voxels, that corresponds to a $3 \mathrm{D}$ volume of $40 \times 40 \times 12 \mu \mathrm{m}^{3}$. The spatial resolution limits of the proposed setup are $\Delta x=\Delta y=\lambda_{0} /\left(\mathrm{NA}_{\mathrm{o}}+\mathrm{NA}_{\mathrm{c}}\right) \approx 200 \mathrm{~nm}$ and $\Delta z=\lambda_{0} /\left(n_{\text {imm }}-\left(n_{\text {imm }}^{2}-\mathrm{NA}_{\mathrm{o}}^{2}\right)^{1 / 2}\right) \approx$ $500 \mathrm{~nm}$, in the traverse and axial direction respectively, where $n_{\text {imm }}=1.518$ is the refractive index of the objective immersion oil (Olympus Type F).

\subsection{2 | RI deconvolution}

The intensity images obtained with the setup from Fig. 1 are described by the convolution of object scattering potential and the microscope OTF itself. A reliable $3 \mathrm{D}$ reconstruction of the object RI can be obtained by performing a direct (non-iterative) Wiener deconvolution algorithm that is described in the frequency domain $\left(\boldsymbol{\rho}=\left(\rho_{x}, \rho_{y}, \rho_{z}\right)\right)$ as follows,

$$
P(\boldsymbol{\rho})=\frac{I(\boldsymbol{\rho}) H_{E}^{*}(\boldsymbol{\rho})}{\left|H_{E}(\boldsymbol{\rho})\right|^{2}+\beta},
$$

where $P$ denotes the scattering potential, $I$ is the measured intensity stack, $H_{E}$ represents the OTF of the microscope and $\beta$ serves as a regularization parameter. Then, the inverse Fourier transform is applied over Eq.(1) and the result is inserted in the following expression,

$$
n^{2}(\mathbf{r})=P(\mathbf{r}) k_{0}^{-2}+n_{m}^{2}
$$


where $n_{m}$ is the RI of the immersion medium (typically water or an aqueous solution) and $k_{0}$ is the wavenumber. Finally, we only take the real part of $n(\mathbf{r})$ as the sample is assumed to be almost transparent. More details regarding this deconvolution approach can be found in Refs. $\frac{[15,1625]}{\text {. }}$

\subsection{3 | Segmentation procedure based on the RI gradient}

For accurate cell structure analysis, first a proper segmentation must be performed to separate the cell from the surrounding medium (background). For this purpose, the RI gradient modulus $(|\nabla n|)$ has been computed:

$$
|\nabla n(\mathbf{r})|=\sqrt{\left(\frac{n_{x+1, y, z}-n_{x-1, y, z}}{\Delta x}\right)^{2}+\left(\frac{n_{x, y+1, z}-n_{x, y-1, z}}{\Delta y}\right)^{2}+\left(\frac{n_{x, y, z+1}-n_{x, y, z-1}}{\Delta z}\right)^{2}} .
$$

By normalizing the gradient modulus to the range $[0,1]\left(\left|\nabla n_{n}(\mathbf{r})\right|=|\nabla n(\mathbf{r})| / \max [|\nabla n(\mathbf{r})|]\right.$ and fixing a low threshold $(\tau)$ for $\left|\nabla n_{n}\right|$ the RI background can be suppressed. In this way, we can define a refined version of the RI (further referred to as $\widehat{n}(r)$ ) as

$$
\widehat{n}(\mathbf{r})= \begin{cases}n(\mathbf{r}), & \left|\nabla n_{n}\right|>\tau \text { and } n(\mathbf{r})>n_{m}, \\ n_{m}, & \text { elsewhere }\end{cases}
$$

where in this case, $\tau=0.01$ has been considered. This RI gradient modulus has been succesfully applied in similar segmentation tasks as it accounts for RI local variations that are different for the background and for sample itself, typically involving little variations in background in comparison with steep changes in the cell [26]28]. By taking into account the gradient along with the RI itself, one gets a better discrimination of cell structures. For instance, regions with low RI and low RI gradient modulus values correspond to the background. Rather, high RI and high RI gradient modulus are associated with fine details (mainly vesicles and organelles dissolved within the cytoplasm). This gradient information can be easily obtained and incorporated to enhance the detection of different sample components.

\subsection{4 | Derivation of dry mass concentration from the RI reconstruction}

As reported in [129]30], for biological samples immersed in an aqueous medium it exists a linear relationship linking the dry mass concentration (or density, further referred to as DMC) of the sample and its RI, as follows:

$$
C(\mathbf{r})=\left(\frac{\widehat{n}(\mathbf{r})-n_{m}}{\alpha}\right),
$$

where $C$ is the DMC, $\alpha$ represents the so-called specific refractive index increment and $n_{m}$ accounts for the surrounding medium RI (in this case is methanol, so $n_{m}=1.333$ at a wavelength of $\lambda=450 \mathrm{~nm}$ and $300 \mathrm{~K}$ according to Ref. [31]). As stated by Barer ${ }^{[30]}$, the main solid components of the cell protoplasm are proteins, lipids and carbohydrates (typically conforming complexes with proteins), and other components (such as amino acids or nucleic acids) whose RI is close to that of proteins. Although this chemical composition is variable, an average value of $\alpha=0.002 \mathrm{dL} / \mathrm{g}$ is widely accepted within the visible range when nucleated cells are considered [127]30]. Moreover, as explained in Ref. ${ }^{[32]}$, the communication between Leishmania and the macrophages is regulated by proteins, so their effect is expected to prevail in the cell RI.

\section{3 | RESULTS AND DISCUSSION}

The samples of DH82 cells were divided into four categories, according to the presence of the parasite (parasited or healthy groups) and the period of time $(24 \mathrm{~h}$ or $72 \mathrm{~h}$ ) from the preparation, and labelled as $\boldsymbol{P} 24$, $H 24, P 72$ and $H 72$ respectively. The four groups have been analyzed with the same microscope shown in Fig. 1 by considering stained and unstained cells separately. 
(a) Healthy $24 \mathrm{~h}$

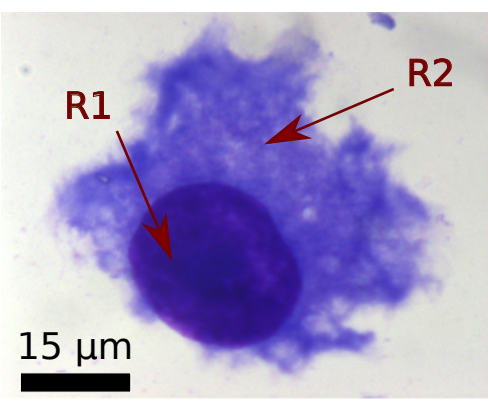

(d) Parasited $72 \mathrm{~h}$

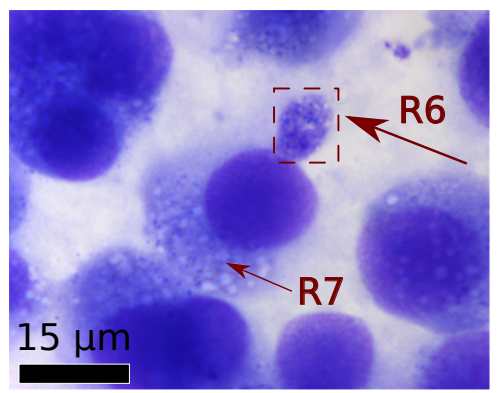

(b) Healthy $72 \mathrm{~h}$

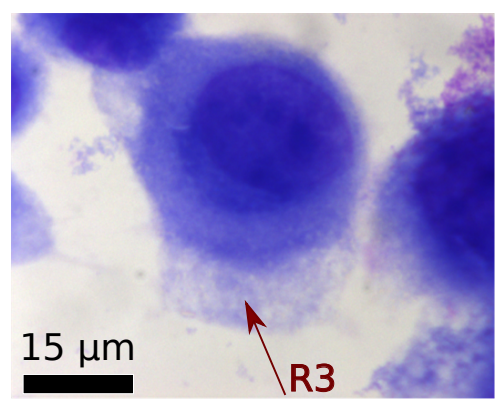

(c) Parasited $24 \mathrm{~h}$
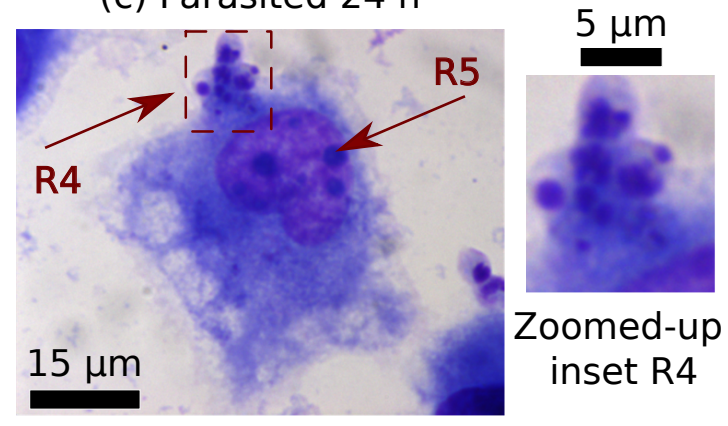

(e) Parasited $72 \mathrm{~h}$


FIGURE 2 DH82 cell images, corresponding to the healthy control (a-b) and parasited groups (c-e). Cells were infected with promastigotes of Leishmania infantum BCN150 at a ratio 10:1 (parasites:cells). After $24 \mathrm{~h}(\mathrm{a}, \mathrm{c})$ or $72 \mathrm{~h}(\mathrm{~b}, \mathrm{~d}, \mathrm{e})$ cells were fixed in methanol, stained with Giemsa and evaluated by optic microscopy (100x magnification, $N A_{c}=1.4$ ). Particular features of the cells are marked with red arrows while a close-up view of the intracellular amastigotes within the dashed boxes have been shown in the insets (c-e).

\section{1 | Stained cells results}

In conventional clinical practice, staining techniques are considered mandatory when it comes to Leishmania study. Straightforward inspection of unstained samples intensity distribution obtained with an optical microscope (e.g. under conventional BFI) does not provide enough contrast to permit the parasite observation. For this reason, firstly stained samples are displayed in Fig. 2 , that have been acquired with a conventional CMOS camera (Thorlabs DCC1240C, exposure time $10 \mathrm{~ms}$ ). Note that either the nucleus, some intracellular structures and the parasites are dyed by Giemsa in a purple bluish color.

Healthy DH82 cells (see Fig.2 (a)) are composed of a big nucleus (R1, dyed in dark purple) that occupies most of the cell volume (R2). Moreover, some samples present recognizable membrane projections (see R3 in Fig.2 (b)) regulated by proteins (particularly actin) that they need to perform phagocytosis.

After applying Giemsa stain to infected samples, one is able to recognize the Leishmania into the apoptosis blebs (see R4 in Fig. 2 (c)) and other intranuclear inclusions immersed within the nucleus plasma (see R5). Particularly, these blebs or membrane extrusions are used for the egress of amastigotes from the host cell to neighbouring cells. In this way, other cells uptake these parasites and the infection is spread. The apoptotical structures appear into the 24-72 $\mathrm{h}$ after the infection, as also shown in regions R6-R7 in Fig. 2 (d). Indeed, parasited cells manifest certain amastigotes located within the cytoplasm (see R8-R9 in Fig. 2 (e)) that have been engulfed by the host. Once stained, the parasites identification can also be achieved by identifying certain structures, such as their nuclei (see R10) or their kinetoplast (typically rounded shaped). Other studies have also reported morphological changes between parasited and healthy macrophages like a certain cell shrinkage that takes place as the infection progresses, see for example ${ }^{[33]}$. In this way, the staining procedure helps to localize the parasite and some cell structures providing qualitative information. Conversely, in this work we consider a quantitative characterization of the cell by analyzing its RI and its DMC along with their temporal evolution in order to obtain information about the parasite spreading in the host cell. 

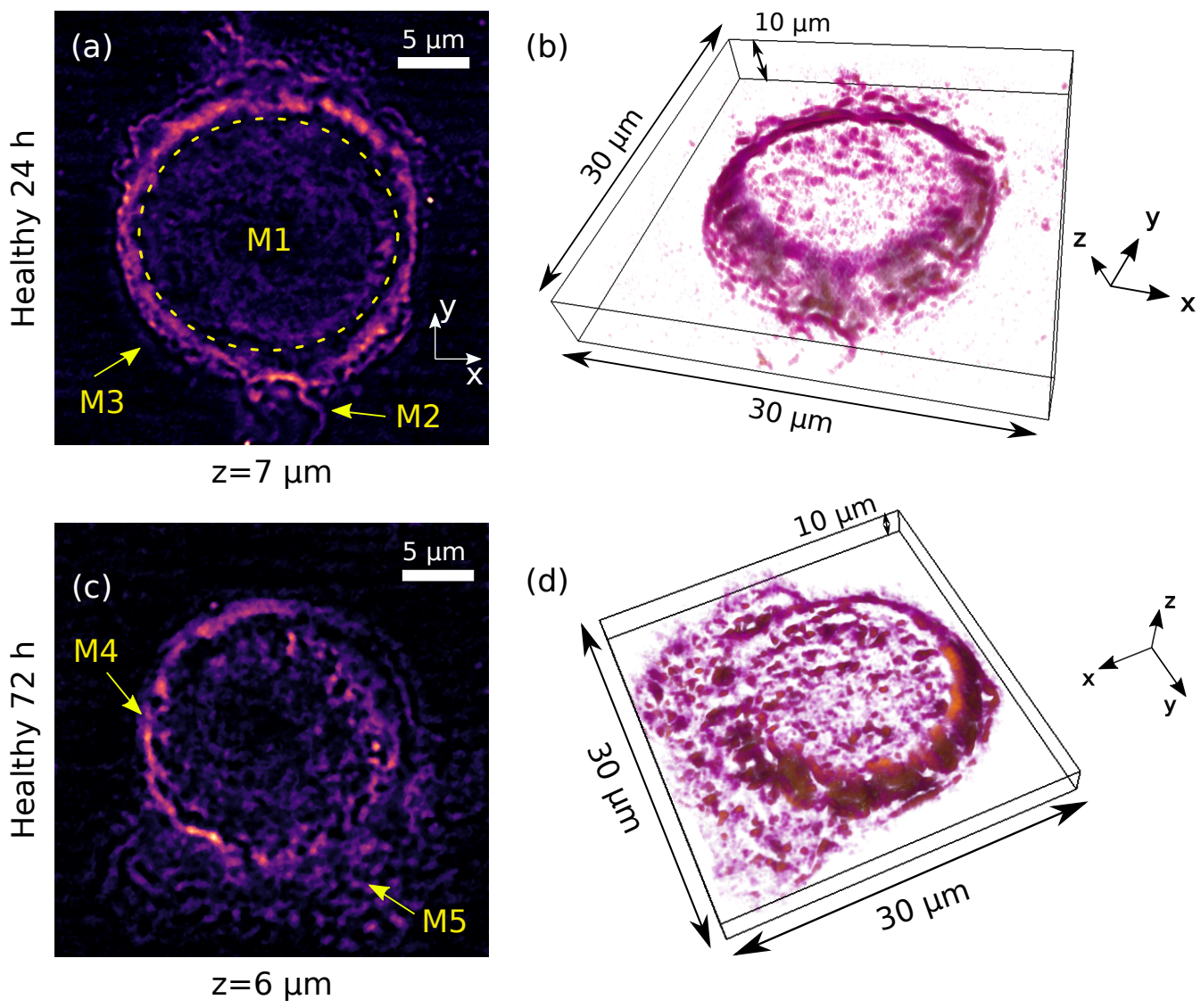

(d)



RI (a.u)

$\operatorname{DMC}(g / d l)$

1.333

1.361



FIGURE 3 Results of healthy DH82 samples. 2D xy-slices of the RI distribution for $\boldsymbol{H} 24$ and $\boldsymbol{H} 72$ macrophages are shown in (a) and (c) respectively, while the corresponding 3D DMC reconstructions are displayed in (b, d). The boxes in (b, d) represent the same volume of $30 \times 30 \times 10 \mu^{3}$.

\subsection{Analysis of unstained cells via 3D RI distribution}

To study the modifications induced by the parasite in the macrophages, we analyze unstained samples by using their 3D RI distributions reconstructed via PC-ODT technique. In Fig. 3 (a,c) it has been displayed two xy-slices of the RI corresponding to healthy macrophages belonging to $H 24$ and $H 72$ groups respectively, while Fig. 3 (b,d) show the whole studied 3D stack of these samples. Specifically, one identifies characteristic cell structures such as a rounded nucleus (M1 region within the dashed line circle), long-thin pseudopods (M2), fine cell membrane (M3), nuclear envelope (M4) and the typical surface roughness of membrane projections (M5), which have also been recognized in 2D stained images shown in Fig.2 (b). The RI of the macrophage nucleus membrane reaches a value of 1.36 (colored in orange in Fig. 3 ) while that of the cell wall is lower (around 1.35, in purple). These RI values are in good agreement with those previously reported in the literature (in the range $1.35-1.375)^{[2]}$. Apart from this structural information, the reconstructed 3D RI can also provide quantitative data such as the cell DMC during the course of infection. According to Eq. 5, the RI and DMC are linearly related. Therefore, in order to help the visualization of cell DMC we have applied a similar colorbar as in the case of the RI, see Fig. 3 (b, d). Let us underline that this representation has been created by using a open-source software, Drishti imaging suite [28]. The inspection of 3D RI volume enables to determine local variations of its DMC. It was found that the DMC values in the nuclear membrane (above $12 \mathrm{~g} / \mathrm{dL}$ ) are significantly larger than in the rest of the structure (3$3.5 \mathrm{~g} / d L$ ) for both healthy groups. From this preliminary analysis we conclude that no relevant differences between healthy cells $H 24$ and $H 72$ arise with regard to their RI and DMC. 

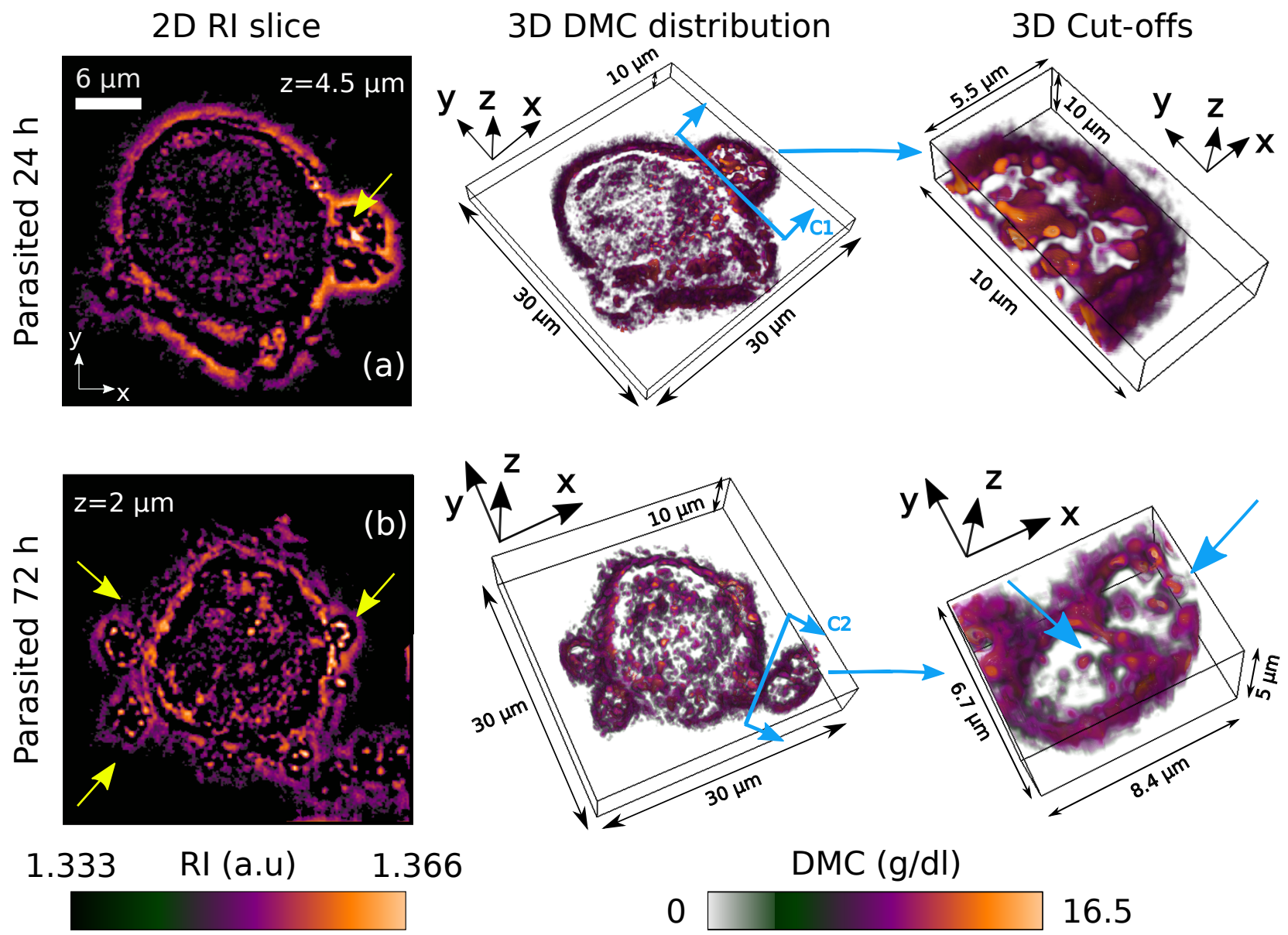

DMC $(g / d l)$

0 16.5

FIGURE 4 Results of parasited DH82 samples, for $P 24$ (a) and $P 72$ (b). For each cell three graphs are included: a 2D xy-slice of the RI (first column, in which yellow arrows point towards apoptosis blebs), a view of the 3D DMC reconstruction (second column) and the corresponding magnified regions of the latter (third column) cut by planes $\mathrm{C} 1$ and $\mathrm{C} 2$ respectively, for highlighting the location of some parasite amastigotes within the membrane blebs.

In the case of DH82 cells infected by Leishmania, the most remarkable difference in their structure lies in cellular fragmentation and presence of apoptotic blebs (as indicated by yellow arrows in Fig. 4 ) that do not appear in healthy samples. These apoptosis evidences can appear as early as $24 \mathrm{~h}$ after the infection (see Fig. 4 (a)) and have also been appreciated in the bright-field intensity images of the stained cells displayed in Fig. 2 (c-d). For instance, from the RI is possible a direct detection of intracellular amastigotes exhibiting high RI values (above 1.36, colored in orange in the first column of Fig. 4 ) and located within the blebs. We recall that such blebs are used for spreading the parasitical infection to neighbour cells.

Nevertheless, the reconstructed RI maps also enable a quantitative estimation of the modifications induced by the parasite such as DMC changes in certain cell regions and its evolution as the infection progresses. Moreover, the DMC (see Fig.4, second and third columns) of parasited macrophages nucleus membrane (between $14-15 \mathrm{~g} / d L$ ) is higher than that of the healthy samples (between $12-13 \mathrm{~g} / d L$ ). Again, as in healthy samples, the DMC of nucleus membrane is superior to the one from the rest of infected cell structure. The rise of average DMC in parasited samples in comparison with the healthy ones can be explained because apoptotic bodies (that typically contain nuclear material and actin cytoskeleton fragments) are shed within the cell ${ }^{[34]}$, thus changing its DMC.

Let us now analyzing the temporal evolution of the mean DMC, 24 and $72 \mathrm{~h}$ after infection. It has been considered 40 cells from each group ( $H 24, H 72, P 24$ and P72) and their average DMC has been calculated (see Fig.5 ). Relevant differences emerge between the infected and control groups. Both uninfected samples 


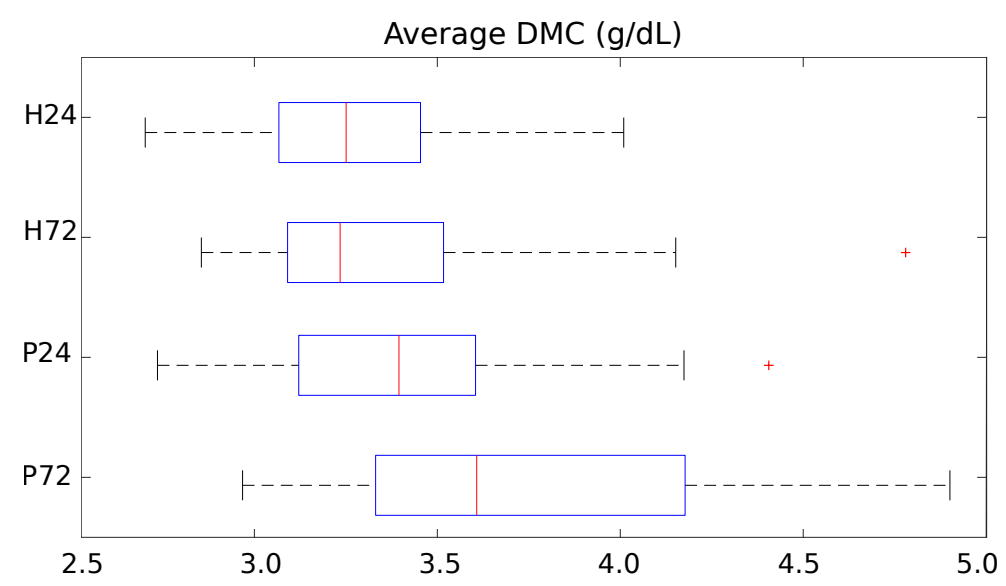

FIGURE 5 Boxplot of the average DMC for the four groups of samples: H24 (healthy 24h), H72 (healthy 72h), P24 (parasited 24h) and P72 (parasited 72h). The red line is the median DMC value and outliers are marked with red crosses.

show similar ranges of averaged DMC: $H 24$ presents DMC values of 2.639-3.947 $\mathrm{g} / \mathrm{d} L$ (median of 3.189 $\mathrm{g} / d L$ ) whereas $H 72$ exhibits DMC of $2.793-4.717 \mathrm{~g} / \mathrm{dL}$ (median of $3.172 \mathrm{~g} / \mathrm{dL}$ ). Nevertheless, when the $P 24$ group is considered, a slight rise in median DMC value $(3.383 \mathrm{~g} / d L)$ is observed in comparison with any of healthy cases. This increment is even more noticeable for $P 72$ cells, for which DMC reaches a median value of $3.595 \mathrm{~g} / \mathrm{dL}$ and it spans over the interval $2.956-4.889 \mathrm{~g} / \mathrm{dL}$. This fact suggests that a DMC increase is associated with the presence of Leishmania and this change is more remarkable as the time since infection increases.

In order to statistically validate this conclusion, also pointed out by the visual inspection of 3D DMC reconstructions, two hypothesis must be validated. First, we need to test whether the healthy groups DMC ( $H 24$ and H72) significantly differs from that of parasited samples (P24 and P72). Secondly, we check if the course of the infection induces changes in DMC by comparing $P 24$ and $P 72$ groups with each other. Each of these statements have been corroborated by conducting paired sample $t$-tests over DMC data, as summarized in Table 1 . Taken altogether, the results confirm that average DMC can be applied as a reliable parameter for analyzing both the health state of the host cell and the development of Leishmania infection.

\section{4 | CONCLUSIONS}

The cell refractive index is a biophysical parameter that represents the intracellular mass and its concentration providing important insight for the study, diagnosis and assessment of cell health state. In this work we have performed a quantitative analysis of both healthy and Leishmania infected macrophages, based on a novel RI tomography PC-ODT technique. We have demonstrated that the cell 3D RI distribution is useful to detect relevant morphological changes in the infected cells such as apoptosis blebs. Our main finding is that the cell dry mass concentration (directly obtained from the cell RI) reveals key quantitative information required to gain knowledge about the Leishmania spreading. It allows reliable detection and temporal

TABLE 1 Results from statistical $t$-tests carried out to verify the relationship between DMC changes and Leishmania presence in DH82 cells.

\begin{tabular}{lcc}
\hline Test & Significant differences? & $\boldsymbol{p}$-value \\
\hline $\boldsymbol{H} 24$ vs $\boldsymbol{P} 24$ & Yes & 0.0214 \\
$\boldsymbol{H} 72$ vs $\boldsymbol{P} 72$ & Yes & 0.0026 \\
$\boldsymbol{P} 24$ vs $\boldsymbol{P} 72$ & Yes & 0.0048 \\
\hline
\end{tabular}


monitorization of the parasite proliferation into the cell volume. According to the experimental results, noninfected cells do not significantly change their DMC between 24-72 $\mathrm{h}$ from the preparation. Conversely, the infected cells exhibit higher mean DMC than the healthy cultures and this fact becomes even more evident as infection progresses. These experimental results are in good agreement with the known life cycle of the Leishmania infantum and its proliferation into the host macrophages. Thus, in comparison with a merely qualitative study over a stained sample, the proposed label-free technique based on RI tomography provides a valuable quantitative measurement to characterize the infection and its temporal evolution.

Note that throughout this work the sample is fixed but it is also possible to apply PC-ODT technique over live cells as reported in [16]. In this way, PC-ODT is appropriate for better taking advantage of temporal changes induced by the parasite in the cell RI. We envision that the increase in DMC could be further exploited to anticipate the development of Leishmania infection. For instance, it could be applied in the assessment of antileishmanial drug efficacy or even integrated in a medical diagnosis protocol. The considered PC-ODT technique is inherently compatible with a conventional wide-field microscope and allows for straightforward and fast RI tomography (typically at a video rate of $10 \mathrm{fps}$ ), which are crucial advantages demanded in the clinical diagnosis protocols.

\section{Disclosures}

The authors declare that there is no conflict of interest.

\section{Acknowledgements}

The Ministerio de Economía y Competitividad is acknowledged for funding the project TEC2014-57394-P. This research was partially supported through a Santander-UCM 2018 project (PR75/18-21567) and by a grant from Comunidad de Madrid (Spain) PLATESA (S2013/ABI-2906). Alicia Mas was supported by a fellowship from Complutense University of Madrid-Santander.

\section{ORCID}

Tatiana Alieva https://orcid.org/0000-0002-7638-9150

José A. Rodrigo https://orcid.org/0000-0001-7313-7313

Juan M. Soto https://orcid.org/0000-0002-7407-2723

Gustavo Domínguez-Bernal https://orcid.org/0000-0002-7693-653X

\section{SUPPORTING INFORMATION}

The following supporting information is available as part of the online article:

Video S1. Rotation of the 3D DMC stack of a DH82 cell after $72 \mathrm{~h}$ of infection by Leishmania infantum. Apoptosis blebs with no membrane rupture confirm the disease.

\section{GRAPHICAL ABSTRACT TEXT}

In this work, a label-free technique for providing a reliable analysis of macrophages infected by Leishmania infantum is presented. It is based on 3D refractive index distribution reconstruction closely related to the estimation of cell volume and dry mass concentration. Relevant differences between uninfected and infected macrophage groups have been found. This approach provides an attractive alternative to conventional staining procedures, thus simplifying the sample preparation. 




\section{GRAPHICAL ABSTRACT FIGURE}

\section{References}

[1] K. G. Phillips, S. L. Jacques, O. J. T. McCarty, Phys. Rev. Lett 2012, 109 (11), 118105.

[2] A. E. Ekpenyong, S. M. Man, S. Achouri, C. E. Byant, J. Guck, K. J. Chalut, J. Biophotonics 2013, 6 (5), 393-397.

[3] T. Kim, R. Zhou, M. Mir, S. D. Babacan, P. S. Carney, L. L. Goddard, G. Popescu, Nat. Photonics 2014, 8 (3), 256-263.

[4] T. A. Zangle, M. A. Teitell, Nat. Methods 2014, 11 (12), 1221-1228.

[5] J. Yoon, K. Kim, H. Park, C. Choi, S. Jang, Y. Park, Biomed. Opt. Express 2015, 6 (10), 3865-3875.

[6] S. Lee, H. Park, K. Kim, Y. Sohn, S. Jang, Y. Park, Sci. Rep. 2017, 7 (1), 1039.

[7] W. Hsu, J. Su, C. Chang, K. Sung, in Optics in Health Care and Biomedical Optics V, International Society for Optics and Photonics, 2012, p. 855310.

[8] B. Simon, M. Debailleul, A. Beghin, Y. Tourneur, O. Haeberlé, J. Biophotonics 2010, 3 (7), $462-467$.

[9] Y Park, M Diez-Silva, G Popescu, G Lykotrafitis, W Choi, M S Feld, S Suresh, Proc. Natl. Acad. Sci. U.S.A. 2008, 105 (37), 13730-13735.

[10] K. Kim, H. Yoon, M. Diez-Silva, M. Dao, R. R. Dasari, Y. Park, J. Biomed. Opt 2013, 19 (1), 011005.

[11] Y. Cotte, F. Toy, P. Jourdain, N. Pavillon, D. Boss, P. Magistretti, P. Marquet, C. Depeursinge, Nat. Photonics 2013, 7 (2), 113-117.

[12] A.V. Belashov, A.A. Zhikhoreva, V.G. Bespalov, O.S. Vasyutinskii, N.T. Zhilinskaya, V.I. Novik, I.V. Semenova, Tech Phys Lett 2017, 43 (10), 932-935.

[13] Y Sung, W Choi, C Fang-Yen, K Badizadegan, R R Dasari, M S Feld, Optics express 2009, 17 (1), $266-277$.

[14] M. Chen, L. Tian, L. Waller, Biomed. Opt. Express 2016, 7 (10), 3940-3950.

[15] J. M. Soto, J. A. Rodrigo, T. Alieva, Opt. Express 2017, 25 (14), 15699-15712.

[16] J. A. Rodrigo, J. M. Soto, T. Alieva, Biomed. Opt. Express 2017, 8 (12), 5507-5517.

[17] J. Dujardin, L. Campino, C. Cañavate, J. Dedet, L. Gradoni, K. Soteriadou, A. Mazeris, Y. Ozbel, M. Boelaert, Emerging Infect Dis 2008, 14 (7), 1013.

[18] M. Podinovskaia, A. Descoteaux, Future Microbiol 2015, 10 (1), 111-129.

[19] World Health Organization, Leishmaniasis. Key facts., Available from: http://www.who.int/ news-room/fact-sheets/detail/leishmaniasis [last accessed October 2018]. 
[20] R. Kumar, C. Engwerda, Clin Transl Immunology 2014, 3 (3), e13.

[21] J. F. Barbosa, S. M. de Figueiredo, F. M. Monteiro, F. Rocha-Silva, C. Gaciele-Melo, S. SC Coelho, S. Lyon, R. B. Caligiorne, Recent Pat Endocr Metab Immune Drug Discov. 2015, 9 (2), 90-102.

[22] M. L. Wellman, S. Krakowka, R. M. Jacobs, G. J. Kociba, In Vitro Cell. Dev. Biol.-Animal 1988, 24 (3), 223-229.

[23] J. Fernández-Cotrina, V. Iniesta, S. Belinchón-Lorenzo, R. Muñoz-Madrid, F. Serrano, J. C. Parejo, L. Gomez-Gordo, M. Soto, C. Alonso, L. C. Gómez-Nieto, Vet Parasitol. 2013, 192 (1-3), 118-128.

[24] G. Domínguez-Bernal, M. Jiménez, R. Molina, L. Ordóñez-Gutiérrez, A. Martínez-Rodrigo, A. Mas, M. T. Cutuli, J. Carrión, Parasit Vectors 2014, 7 (1), 499.

[25] J. M. Soto, J. A. Rodrigo, T. Alieva, Opt. Lett. 2018, 43 (19), 4699-4702.

[26] G. Li, T. Liu, A. Tarokh, J. Nie, L. Guo, A. Mara, S. Holley, S. T. C. Wong, BMC Cell Biol. 2007, 8 (1), 40.

[27] A. Yakimovich, R. Witte, V. Andriasyan, F. Georgi, U. F. Greber, mSphere 2018, 3 (6), e00599-18.

[28] A. Limaye, in Developments in X-Ray Tomography VIII, International Society for Optics and Photonics, 2012, p. 85060X.

[29] D. M. Mahlmann, J. Jahnke, P. Loosen, Eur J Phycol. 2008, 43 (4), 355-364.

[30] R. Barer, Nature 1953, 172 (4389), 1097-1098.

[31] K. Moutzouris, M. Papamichael, S. C. Betsis, I. Stavrakas, G. Hloupis, D. Triantis, Appl. Phys. B 2014, $116(3), 617-622$.

[32] J. M. Silverman, J. Clos, C. C. de Oliveira, O. Shirvani, Y. Fang, C. Wang, L. J. Foster, N. E. Reiner, J. Cell Sci. 2010, 123, 842-852.

[33] S. Gannavaram, A. Debrabant, Front Cell Infect Microbiol 2012, 2, 95.

[34] F. Real, P. T. V. Florentino, L. C. Reis, E. M. Ramos-Sanchez, P. S. T. Veras, H. Goto, R. A. Mortara, Cell Microbiol 2014, 16 (10), 1549-1564.

How to cite this article: J. M. Soto, J. A. Rodrigo, A. Mas, G. Domínguez-Bernal, and T. Alieva (2019), Label-free bioanalysis of Leishmania infantum using refractive index tomography with partially coherent illumination, J. Biophysics, 2019;00:1-6. 